\title{
Bagging bursts, swiftly
}

\section{The gamma rays spat out by dying stars last an instant. Tony Reichhardt reports on the fast-response satellite that hopes to capture them.}

$\mathrm{T}$ en years ago, science fiction could get away with speculating that gammaray $(\gamma$-ray) bursts were the exhaust trails of alien spaceships. And why not? No one had a sure explanation for these mysterious high-energy flashes, which briefly outshine everything in the $\gamma$-ray sky, and just as mysteriously flicker out, never to reappear.

But the truth is equally dramatic. Most astronomers now believe these bursts are the dying gasps of massive imploding stars. Still, not all the mysteries have been solved. So a new NASA satellite called Swift is on the case, with launch scheduled for 8 November from Cape Canaveral in Florida.

Swift is not an acronym but a design philosophy. Speed is critical for studying $\gamma$-ray bursts, some of which last only a few milliseconds. The new satellite will be better than previous spacecraft at detecting the bursts, relaying their coordinates quickly to groundbased telescopes, then swivelling its own $\mathrm{X}$-ray and optical/ultraviolet instruments to study the burst 'afterglow' that shines albeit not as brightly - in other wavelengths.

This afterglow is key, because it tells observers how much energy is emitted at different stages of the explosion, and provides them with clues to its origins. There is a consensus regarding the source of longerlasting $\gamma$-ray bursts, but the shorter bursts of less than two seconds remain mysterious, as no afterglow has been seen at any other wavelength. The team behind Swift hopes to fix that.

First noticed in the 1960s, $\gamma$-ray bursts are going off all the time, all over the sky. The Compton Gamma Ray Observatory catalogued more than 1,600 in the 1990 s, but wasn't able to settle the key question of how far away they were. Then, in 1997, the Italian-Dutch spacecraft BeppoSAX saw an Xray afterglow immediately following a burst. Optical afterglows were soon detected for other bursts, and these multi-wavelength observations confirmed that $\gamma$-ray bursts were very far away, in galaxies other than our own.

Theorists had trouble explaining how objects so distant could produce such

data for the short bursts, and Swift should oblige them.

But classifying bursts as long or short doesn't capture their full diversity. Astronomers keep finding oddball $\gamma$-ray bursts with lower-than-typical brightness, or unusual energy distributions, that muddy the theoretical waters (see Nature 430, 623; 2004). This leads veteran bursthunters such as Gerald Fishman of NASA's Marshall Space Flight Center in Huntsville, Alabama, to argue that it's naive to try fitting all $\gamma$-ray bursts into two neat categories. "I definitely think we're in for some big surprises with Swift," he says.

Swift will have better speed, accuracy and sensitivity than today's best burst-finders: NASA's High Energy Transient Explorer (HETE-2) and Europe's International Gamma-Ray Astrophysics Laboratory (INTEGRAL). Swift's Burst Alert Telescope, with its large array of cadmium-zinc telluride detectors, will be the most sensitive astonishing amounts of energy — akin to our Sun's total lifetime output - in just a few seconds. But they reasoned that dying massive stars would be likely to expel jets of material at nearly the speed of light. The overall explosion didn't need to be so powerful if the energy was concentrated in jets shooting out from the star's poles. However, this means that we only see the small fraction of bursts whose jets happen to point towards Earth.

\section{The long and the short of it}

Further observations of $\gamma$-ray bursts and their afterglows led astrophysicists to lump these exotic objects into two categories. First and most common are the long bursts (more than two seconds) produced by large stellar explosions (hypernovae). The resulting 'collapsars' shoot out high-energy jets, although physicists disagree on whether the $\gamma$-rays come from the jets themselves or from a shell of material expanding rapidly away from the scene of the disaster.

Less common are the unexplained short bursts. These might represent a different type of collapsar, or they may be due to binary systems crashing into each other - a neutron star merging with another neutron star or a black hole. Scientists who model the physics of bursts would dearly love to have multispectral $\gamma$-ray imager ever flown. Mission scientists estimate they'll see between 100 and 150 bursts a year - although the number could climb higher because Swift will see fainter objects than previous telescopes have done. Astronomers hope it will smoke out a population of low-energy bursts that have escaped detection byless sensitive satellites.

They also expect the high-resolution images obtained by Swift's X-ray and opti$\mathrm{cal} /$ ultraviolet instruments to yield clues to burst origins. For nearby bursts, Swift should be able to pinpoint both the host galaxy and where in the galaxy the explosion occurred. If short bursts are located mainly in starforming regions, that would favour the collapsar model, because hypernovae tend to be very young stars, unlike the much older neutron stars or black holes of binary systems.

Swift will also immediately relay precise coordinates for the burst to Earth, where dozens of observers - professional and amateur - will be poised to follow up. It should be possible to get telescopes viewing the scene within an hour, when the critical early afterglow will still be in progress. And this powerful combination of ground- and space-based observations, Fishman says, "will take us into uncharted waters".

Tony Reichhardt writes for Nature from Washington DC. 